\title{
Evolución de la Tasa de Mortalidad Neonatal en la Región Amazonas, Perú, 2005 - 2018
}

Evolution of the Neonatal Mortality Rate in the Amazon Region - Peru, 2005 - 2018

\section{RESUMEN}

\author{
Mariel Del Rocío Chotón Calvo', Luis Felipe Gonzales Llontop ${ }^{2}$
}

Se realizó un estudio descriptivo longitudinal, con el objetivo de analizar la tendencia de la mortalidad neonatal, teniendo en cuenta el sexo, lugar de parto y las principales causas de defunción entre los años 2005 al 2018 en la Región Amazonas, para lo cual se tomó información de procedente de la Dirección Regional de Salud (DIRESA) de Amazonas, no contando con información de defunciones neonatales de años anteriores al 2010. Los resultados muestran que durante los años 2006 al 2018 ocurrieron 34582 nacimientos y durante los años 2010 al 2018 ocurrieron 727 defunciones de neonatos en la Región Amazonas; además la Tasa de mortalidad neonatal (TMN) para el período 2010-2018 presentó tendencia descendente, reportándose una TMN de 44,29\%o en el año 2012 y de $15,51 \%$ en el año 2018 es decir que en ese año fallecieron aproximadamente 16 neonatos por cada 1000 recién nacidos vivos. Teniendo en cuenta el sexo de los neonatos en el año 2018 la TMN fue mayor en varones $(21,44 \%$ ) y menor en mujeres $(9,79 \%)$, y mayor en partos institucionales $(19,60 \%)$ que en partos domiciliarios $(11,63 \%)$; asimismo teniendo en cuenta las causas básicas de muerte neonatal se tuvo que en el año 2018 la TMN fue de $13,79 \%$ debido a asfixia y causas relacionadas, de $22,98 \%$ debido a infecciones, de 4,60\% debido a malformación congénita letal, de $23,14 \%$ debido a prematurez-inmaturidad, y de 27,58\% debido a otras causas, concluyéndose que las TMN experimentaron descensos significativos, presentando tendencia decreciente durante el período de estudio.

Palabras clave: mortalidad neonatal, tasas, tendencia.

\begin{abstract}
A longitudinal descriptive study was carried out, with the aim of analyzing the trend of neonatal mortality, taking into account sex, place of birth and the main causes of death between 2005 and 2018 in the Amazon Region, for which it was taken information from the Regional Directorate of Health (DIRESA) of Amazonas, not having information of neonatal deaths of years prior to 2010. The results show that during the years 2006 to 2018 there were 34582 births and during the years 2010 to 2018 there were 727 deaths of neonates in the Amazon Region; In addition, the neonatal mortality rate (NTM) for the 2010-2018 period showed a downward trend, with a NTM of $44.29 \%$ in 2012 and $15.51 \%$ in 2018, which means that approximately 16 died in that year. neonates per 1000 live newborns. Taking into account the sex of neonates in 2018, the TMN was higher in men (21.44\%) and lower in women $(9.79 \%$ ), and higher in institutional deliveries (19.60\%) than in home deliveries (11.63\%o); also taking into account the basic causes of neonatal death, in 2018 the TMN was $13.79 \%$ due to asphyxiation and related causes, $22.98 \%$ due to infections, $4.60 \%$ due to congenital malformation lethal, of $23.14 \%$ due to prematurityimmaturity, and of $27.58 \%$ due to other causes, concluding that the TMN experienced significant decreases, presenting a decreasing trend during the study period.
\end{abstract}

Keywords: neonatal mortality, rates, trend.

'Profesora Asociada de la Facultad de Ingeniería de Sistemas y Mecánica Eléctrica, Universidad Nacional Toribio Rodríguez de Mendoza de Amazonas.marielchoton@untrm.edu.pe

${ }^{2}$ Profesor Asociado de la Facultad de Educacación y Ciencias de la Comunicacon, Universidad Nacional Toribio Rodríguez de Mendoza de Amazonas.luisfego@untrm.edu.pe 


\section{INTRODUCCIÓN}

En una población, la mortalidad constituye uno de los principales determinantes del cambio demográfico porque no sólo determina su crecimiento poblacional, sino que también es utilizada en el cálculo de indicadores que son sumamente importantes para el sector salud, así como también lo es para la planeación, ejecución, evaluación y gestión integral de las políticas de gobierno. La mortalidad está relacionada a factores producidos por las enfermedades, por la interacción con el medio ambiente, y con el desarrollo tecnológico de un país, evidenciando la situación de las condiciones socioeconómicas en las que vive una población.

Es así que el estudio de la mortalidad es de suma importancia en una población, sobretodo aquella ocurrida durante el primer año de vida, la cual ha sido asociada con las condiciones de bienestar, al nivel social y cultural familiar, las cuales condicionan el acceso a los servicios de salud, agudizando aúna más el riesgo de la vulnerabilidad que tienen los recién nacidos vivos con los factores patógenos de la madre y del medio ambiente que la rodea.

Durante muchos años, se ha estudiado la mortalidad infantil debido a que constituye un indicador del nivel socioeconómico de un país, condiciones de vida de una población, además de ser un buen indicador sanitario, por tal motivo se ha descompuesto la mortalidad infantil para un mejor estudio, en mortalidad post neonatal (entre 28 y menos de 1 año de vida), y la mortalidad neonatal (menor de 28 días) en sus dos componentes: precoz (menos de 7 días) y tardía (de 7 a 28 días), resultando que también estos indicadores se utilizan también para medir la calidad de vida la población, tanto es así que algunos autores manifiestan que la mortalidad neonatal resultaría ser un buen indicador para medir la calidad de las atenciones recibidas durante el periodo natal, recomendándose el análisis periódico de estos indicadores para un análisis a traves del tiempo.

Las estimaciones acerca de la mortalidad infantil permite identificar aspectos claves, tales como el avance en el control de las principales causas de muerte, los cambios en los patrones epidemiológicos que surgen como respuesta de los esfuerzos y de los estudios procedentes del sector salud.

A nivel mundial las tasas de mortalidad infantil han mostrado un descenso significativo, sobretodo en países desarrollados donde las condiciones de vida son mejores que en los paísis en vías de desarrollo, en los cuales aún estos indicadores muestran cifras alarmantes, considerando que las causas más frecuentes son debido a factores endógenos los cuales deben estar controlados en una población. En el Perú también se ha mostrado un descenso significativo en los últimos años, pasando de 40,9\%o en el año 2000 a $34,3 \%$ en el año 2005, y de 29,1\%o al 2010.

Según la OMS, pese a los progresos en supervivencia infantil, cada día mueren 7000 recién nacidos vivos, y de mantenerse esa tendencia, 30 millones de recién nacidos morirán durante sus primeros 28 días de vida entre los años 2017 y 2030.

En los últimos 20 años, en el Perú, la mortalidad infantil y la mortalidad neonatal han descendido significativamente, constituyendo uno de los principales logros sanitarios. Actualmente la proporción de muertes de recién nacidos constituye el principal componente de la mortalidad de menores de un año. La mortalidad neonatal es un evento de salud pública de notificación obligatoria y su vigilancia en el país está basada en la Norma Técnica Sanitaria 078-MINSA/DGE3 que establece el Subsistema Nacional de Vigilancia Epidemiológica Perinatal y Neonatal, en el cual se incluye la vigilancia de las muertes fetales y neonatales y la Directiva Sanitaria Directiva Sanitaria N046-MINSA/DGE-V.01. Es así que la mortalidad neonatal se redujo en $67 \%$ desde el año 1995; sin embargo la proporción de mortalidad neonatal frente a la mortalidad infantil se mantiene estacionario en el tiempo, representando el 52,9\% de la mortalidad infantil, frente al $49 \%$ que tenía el año 1992.

Los resultados del presente estudio están orientados a proporcionar información de la tendencia de la mortalidad neonatal (TMN) en recién nacidos vivos de la Región Amazonas-Perú y conocer su valor numérico, lo cual permite analizar su tendencia, constituyendo una herramienta indispensable para la adecuada toma de decisiones en cuanto a la orientación en la realización de que una adecuada planificación de la gestión de los servicios de salud para el recién nacido y la gestante, la priorización de los principales problemas de salud que afectan a las poblaciones más vulnerables.

\section{MATERIAL Y MÉTODOS}

Tipo y diseño de investigación: Descriptiva, longitudinal de tendencia y observacional. , cuyo según diseño es:

$\begin{array}{ccccccc}t_{1} & t_{2} & t_{3} & \cdot & \cdot & . & t_{n+l} \\ N G_{x l} & \boldsymbol{O}_{l} & \boldsymbol{O}_{2} & \cdot & \cdot & . & \boldsymbol{O}_{n}\end{array}$


Población: Estuvo constituida por el número total de nacimiento registrados en la Oficina de Estadística y Sistemas de salud, BD Hechos Vitales de la Dirección Regional de Salud (DIRESA) de Amazonas, durante los años 2005 al 2018. Así como también estuvo constituida por el número total de defunciones de neonatos registrados por la Oficina de Epidemiología de la DIRESAAmazonas, durante los años 2005 al 2018.

Muestra: Estuvo constituida por el número total de nacimientos y defunciones neonatales registrados por la DIRESA Amazonas, durante los años 20102018 mostrados en la siguiente tabla:

Tabla 1: Número de nacimientos ocurridos en la Región Amazonas. Años 2006-2018.

\begin{tabular}{cccc} 
Años & Masculino & Femenino & Total \\
\hline 2010 & 295 & 299 & 594 \\
2011 & 1454 & 1436 & 2890 \\
2012 & 885 & 831 & 1716 \\
2013 & 1497 & 1349 & 2846 \\
2014 & 1974 & 1924 & 3898 \\
2015 & 2257 & 2290 & 4547 \\
2016 & 2551 & 2455 & 5006 \\
2017 & 2688 & 2554 & 5242 \\
2018 & 2565 & 2656 & 5221 \\
\hline
\end{tabular}

Fuente: Elaboración propia en base a los registros de la Dirección Regional de Salud Amazonas./ Dirección de Estadística y Sistemas de Salud, BD Hechos vitales.

Tabla 2: Número de defunciones neonatales ocurridas en la Región Amazonas. Años 2005-2018.

\begin{tabular}{|c|c|c|c|c|c|}
\hline \multirow{2}{*}{ Años } & \multirow{2}{*}{} & \multicolumn{2}{|c|}{ Total } & \multicolumn{2}{c|}{ Tipo de parto } \\
\cline { 3 - 6 } & Masculino & Femenino & Domiciliario & Institucional \\
\hline 2010 & 54 & 27 & 27 & 23 & 31 \\
\hline 2011 & 75 & 46 & 29 & 41 & 34 \\
\hline 2012 & 76 & 50 & 26 & 34 & 42 \\
\hline 2013 & 94 & 58 & 36 & 39 & 55 \\
\hline 2014 & 94 & 48 & 46 & 35 & 59 \\
\hline 2015 & 80 & 42 & 38 & 33 & 47 \\
\hline 2016 & 89 & 55 & 34 & 27 & 62 \\
\hline 2017 & 84 & 53 & 31 & 30 & 54 \\
\hline 2018 & 81 & 55 & 26 & 30 & 51 \\
\hline
\end{tabular}

Fuente: Elaboración propia en base a los registros de la Dirección Regional de Salud Amazonas./ Dirección de Epidemiología.

\section{Método, técnica e instrumento}

Se utilizó el método inductivo - deductivo partiendo de hechos singulares a proposiciones generales, apoyados en las aseveraciones y generalizaciones a partir de las cuales se realizaron inferencias particulares. También se aplicó el método analítico sintético, dinámico y continuo porque permitió caracterizar, medir y explicar la tendencia de la mortalidad neonatal en la Región Amazonas, incrementando el conocimiento de la realidad, facilitando la comprensión del objeto de estudio, para lo cual se realizó el análisis de documentos, también la base de datos de la DIRESA, así como también de fuentes bibliográficas relacionadas al tema de investigación, lo cual fue organizado y procesado utilizando la hoja de cálculo Excel para su posterior análisis, calculándose la tasa de mortalidad neonatal (TMN).

Para el cálculo de la tasa de mortalidad neonatal (TMN) se utilizó la siguiente fórmula:

$$
T M N=\frac{\mathrm{N}^{\circ} \text { defunciones de niños menores de } 28 \text { días }}{\mathrm{N}^{\circ} \mathrm{RNV}} x k
$$

\section{Donde:}

- TMN : Tasa de mortalidad neonatal

- K: constante igual de 1000

- RNV: Número de recién nacidos vivos

\section{RESULTADOS}

Tabla 3: Tasa de mortalidad neonatal en la Región Amazonas durante los años 2010-2018.

\begin{tabular}{|c|c|c|c|}
\hline Años & \multicolumn{2}{|c|}{ Masculino Femenino } & Total \\
\hline 2010 & 91.53 & 90.30 & 90.91 \\
\hline 2011 & 31.64 & 20.19 & 25.95 \\
\hline 2012 & 56.50 & 31.29 & 44.29 \\
\hline 2013 & 38.74 & 26.69 & 33.03 \\
\hline 2014 & 24.32 & 23.91 & 24.11 \\
\hline 2015 & 18.61 & 16.59 & 17.59 \\
\hline 2016 & 21.56 & 13.85 & 17.78 \\
\hline 2017 & 19.72 & 12.14 & 16.02 \\
\hline 2018 & 21.44 & 9.79 & 15.51 \\
\hline
\end{tabular}

Fuente: Elaboración propia.

Tabla 4: Tasa de mortalidad neonatal en la Región Amazonas durante los años 2010-2018, según el tipo de parto. 


\begin{tabular}{ccc} 
Años & Domiciliario & Institucional \\
\hline 2010 & 77,44 & 104,38 \\
2011 & 28,35 & 23,49 \\
2012 & 39,33 & 48,45 \\
2013 & 27,10 & 38,34 \\
2014 & 17,96 & 30,27 \\
2015 & 14,52 & 20,68 \\
2016 & 10,71 & 24,59 \\
2017 & 11,35 & 20,51 \\
2018 & 11,63 & 19,60 \\
\hline
\end{tabular}

Tabla 5: Tasa de mortalidad neonatal en la Región Amazonas, según causa básica de muerte. Años 2010-2018.

\begin{tabular}{|l|l|l|l|l|l|l|l|}
\hline Años & $\begin{array}{l}\text { Asf. y } \\
\text { c.r. }\end{array}$ & Asp. & Infec. & Malf. & $\begin{array}{l}\text { Prem. } \\
\text {-inm. }\end{array}$ & $\begin{array}{l}\text { Ot. } \\
\text { causas }\end{array}$ & Total \\
\hline 2010 & 181.82 & 0.00 & 70.71 & 10.10 & 80.81 & 202.02 & 90.91 \\
\hline 2011 & 56.06 & 0.00 & 26.99 & 6.23 & 29.07 & 37.37 & 25.95 \\
\hline 2012 & 62.94 & 3.50 & 66.43 & 24.48 & 31.47 & 76.92 & 44.29 \\
\hline 2013 & 46.38 & 2.11 & 46.38 & 12.65 & 29.52 & 61.14 & 33.03 \\
\hline 2014 & 24.63 & 1.54 & 24.63 & 15.39 & 20.01 & 58.49 & 24.11 \\
\hline 2015 & 17.15 & 2.64 & 17.15 & 5.28 & 23.75 & 39.59 & 17.59 \\
\hline 2016 & 23.97 & 2.40 & 17.98 & 4.79 & 19.18 & 38.35 & 17.78 \\
\hline 2017 & 20.60 & 2.29 & 22.89 & 8.01 & 22.89 & 19.46 & 16.02 \\
\hline 2018 & 13.79 & 0.00 & 22.98 & 4.60 & 24.13 & 27.58 & 15.51 \\
\hline
\end{tabular}

Fuente: Elaboración propia.

\section{DISCUSIÓN}

El periodo neonatal, comprendido entre el momento del nacimiento y los primeros 28 días de edad, es la etapa más vulnerable de la vida del ser humano, y es en esta etapa que se dan los mayores riesgos para su sobrevivencia; muerte, enfermedades, complicaciones, secuelas. La mortalidad neonatal es un indicador que evidencia el nivel de desarrollo que tiene la atención prenatal y del recién nacido en una determinada área geográfica o en un servicio.

La Tabla 3 muestra como resultado que la tasa de mortalidad neonatal (TMN) en la Región Amazonas se redujo significativamente pasando de ser 44,49 en el año 2012 a 15,51 muertes de neonatos por cada 1000 nacidos vivos en el año 2018, mostrándose un descenso lento pero sostenido a través del tiempo. Estos resultados concuerdan con el descenso de la TMN a nivel mundial que se redujo significativamente, pasando de tener 33 muertes por cada 1.000 nacidos vivos en 1990 a 21 en 2012 $(36 \%)$, lo que se traduce en una reducción de las muertes neonatales de 4,6 millones en 1990 a 2,9 millones en 2012.

Estas tendencias de reducción de la mortalidad neonatal en la Región Amazonas también se presenta a nivel de América Latina, que mostró la reducción de la TMN que fue del 55\%, pasando de 33 muertes por cada 1.000 nacidos vivos en 1990 a 10 en 2012, es decir que se registraron para ese año aproximadamente 106 mil defunciones neonatales. Además que esta situación también se observa en nuestro país, donde la TMN se redujo, según ENDES 2012 , en un $67 \%$ y está directamente relacionada al incremento de la cobertura y mejora de la calidad de los cuidados prenatales y la atención del parto institucional. A partir del año 1990 la mortalidad en la niñez, infantil y neonatal se redujeron significativamente en el Perú, constituyendo uno de los principales logros sanitarios. La Mortalidad neonatal en el período 2005 - 2015 se redujo significativamente en un $67 \%$ y representa un $52,9 \%$ de la mortalidad infantil.

La reducción de la TMN para el Perú, para el año 2012 fue de 12 por cada 1000 recién nacidos vivos según ENDES y la tasa de mortalidad neonatal para la Región Amazonas fue de 15 defunciones por cada 1000 recién nacidos vivos, y para los años 2010 al 2015, la tasa de mortalidad neonatal se ha incrementado de manera considerable, teniéndose que por cada 1000 nacidos vivos, una tasa de 11.89 para el año 2010, y de 19.38 para el año 2015; sin embargo comparando la TMN del año 2015 que fue de 19,38, con la del año 2014 que fue de 23,17 se observa una ligera disminución.

Con respecto a la evolución de la tasa de mortalidad neonatal en la Región Amazonas, según el sexo de los neonatos, se muestra en la Tabla 3 que fue decreciente con mayores valores en varones que en mujeres desde el año 2012 con $56,50 \%$ en varones y $31,29 \%$ en mujeres, llegando en el año 2018 a 21,44\%o en varones y $9,79 \%$ en mujeres. Estos resultados concuerdan con lo publicado por el MINSA en el año 2015 para la Región Amazonas, donde se estimaron para el período 2011-2012, 286 muertes neonatales, con una tasa de mortalidad de 14.8 por cada mil nacidos vivos, de los cuales en varones fue de 19.5 y en mujeres fue de 10 , es decir que fue menor en mujeres que en varones.

Con respecto al lugar de parto, los resultados muestran en la Tabla 4 que la evolución de la tasa de mortalidad neonatal en la Región Amazonas fue decreciente desde el año 2012 registrándose mayores valores en parto institucional comparado con el parto domiciliario, registrándose una TMN de $48,45 \%$ en parto institucional y $39,33 \%$ en parto domiciliario en 
el año 2012, y en el año 2018 de 19,60\%o en parto institucional y de $11,63 \%$ en parto domiciliario.

Estos resultados comparados con la información reportada por ENDES que evaluó la TMN en el período del 2011 al 2015, la proporción de partos institucionales se ha incrementado, de $54.2 \%$ a $73.8 \%$ y es que este tipo de parto constituye o forma parte de una estrategia que permitirá prevenir las muertes maternas, y por ende de los neonatos; claro siempre y cuando las instituciones de salud garanticen el adecuado ambiente, equipos, medicamentos, y también el personal capacitado; además hay que considerar que la cobertura de los partos institucionales en la Región Amazonas tenía en ese período $18 \%$ menos que el valor nacional $(91,8 \%)$.

Los resultados mostrados en la Tabla 5 muestran que para el período de estudio se tuvo disminución en la TMN, es así que en el año 2018 se reportó una TMN de $13,79 \%$ debido a asfixia y causas relacionadas, de $0 \%$ debido a aspiración neonatal de leche y alimento regurgitado, de 22,98\% debido a infecciones, de $4,60 \%$ debido a malformación congénita letal, de $24,13 \%$ o debido a prematurez - inmaturidad, y de $27,58 \%$ debido a otras causas. Estos resultados comparados con el año 2012 tuvieron descensos significativos presentando tendencia decreciente durante el período de estudio. Estos resultados concuerdan con el estudio publicado por la DIRESAMINSA en el año 2013, es así que las defunciones neonatales según causas de muerte y peso al nacer, en el año 2012 la prematuridad fue del 29\%, las infecciones del $20 \%$, la asfixia del $16 \%$, el $12 \%$ por malformaciones congénitas y letales, y el $23 \%$ por otras causas. Estos resultados comparados con las defunciones de recién nacidos vivos con peso normal, sólo el $7 \%$ fue por prematuridad, el $22 \%$ por infecciones, el $28 \%$ por asfixia, el $16 \%$ por malformaciones congénitas, el $27 \%$ por otras causas. Esto además evidencia que el $48 \%$ de las muertes neonatales se pudieron evitar, representando un $48 \%$ de muertes evitables.

En conclusión se tiene que para el año 2018 la primera causa de muerte neonatal fue la prematurezinmaturidad con una TMN de 24,13, seguido por infecciones con 22,98 asfixia y causas relacionadas con 13,79 concordando estos resultados con lo reportado a nivel nacional en el 2016 donde se reportó que la primera causa de defunción neonatal fue la relacionada a la prematuridad-inmaturidad $28 \%$, seguida por las Infecciones $23 \%$, malformaciones congénitas $13.4 \%$, asfixia y causas relacionadas a la atención del parto $12 \%$ y otras causas $24 \%$.
En el cálculo de indicadores demográficos es necesario tener en cuenta la omisión, el sub registro y las fuentes de información de donde estos proceden y que muchas veces varían por no considerarse un registro único, tampoco actualizado en lo referente a nacimientos en la cual existe en la misma DIRESA dos fuentes de registros de información: manual y el Sistema de Registro Certificado de Nacimiento Vivo en línea (CNV) reportado a partir del año 2012, las cuales difieren en el registro, y aún se sigue recibiendo información y actualizando la base de datos con nueva información de las diferentes zonas que no reportan los nacimientos ocurridos, sumándose a ello que se cambió de sistema de almacenamiento de información. A todo esto se suma que no existe una cultura de información precisa, oportuna y veraz en la mayoría de instituciones pese a los esfuerzos realizado, así también no existe una conciencia de reportar información por parte de los ciudadanos que muchas veces omiten información y no le toman demasiada importancia en su registro y mucho menos en su uso pertinente que le pueda favorecer en la toma de decisiones para la implementación de políticas de Estado.

\section{CONCLUSIONES}

-En la Región Amazonas la evolución de la tasa de mortalidad neonatal fue a partir del año 2012 con 44,29 y en el año 2018 con 15,51 muertes neonatales por cada 1000 nacidos vivos, mostrándose un descenso lento pero sostenido a través del tiempo.

-La evolución de la tasa de mortalidad neonatal en la Región Amazonas, según el sexo de los neonatos, fue decreciente lenta y sostenida con mayores valores en varones que en mujeres desde el año 2012 con $56,50 \%$ en varones y $31,29 \%$ en mujeres, llegando en el año 2018 a $21,44 \%$ en varones y $9,79 \%$ en mujeres.

-La evolución de la tasa de mortalidad neonatal en la Región Amazonas, según el lugar de parto, fue decreciente desde el año 2012 registrándose mayores valores en parto institucional comparado con el parto domiciliario, registrándose una TMN de 48,45\% en parto institucional y $39,33 \%$ en parto domiciliario en el año 2012, y en el año 2018 de 19,60\%o en parto institucional y de $11,63 \%$ en parto domiciliario.

-La evolución de la tasa de mortalidad neonatal en la Región Amazonas, según principales causas, fue decreciente desde el año 2012 hasta el año 2018, registrándose en el año 2018 una TMN de 13,79\%o debido a asfixia y causas relacionadas, de 22,98\%o debido a infecciones, de 4,60\% debido a malformación congénita letal, de 24,13\% debido a prematurez-inmaturidad, y de $27,58 \%$ debido a otras 
causas.

\section{REFERENCIAS BIBLIOGRÁFICAS}

DANE. (2010). Metodología de estimación de la tasa de mortalidad infantil municipal. 2005 2007. Bogotá - Colombia: Núm 98.

Dirección Regional de Epidemiología del MINSA. (2013). Análisis de Situación de Salud del Perú. Ministerio de Salud. . Lima - Perú: ISBN: 978-9972-820-98-4.

Echevarría, H. (2016). Diseños de investigación cuantitativa en psicología y educación. . Córdoba - Argentina.: Editora UniRio de la Universidad Nacional de Río Cuarto. .

INEI. (2000). Metodologías para el cálculo de los indicadores de mortalidad. Metodologías Estadísticas, 1(8).

INEI. (2007). Perú: Mortalidad infantil y sus diferencias por departamento, provincia y distrito. Instituto Nacional de Estadística e Informática. Lima.

MINSA - Gobierno Regional de Amazonas. (2015). Análisis de la situacipon de Salud de la Región Amazonas. Dirección Regional de Salud Amazonas, Amazonas. Chachapoyas: Depósito Legal en la Biblioteca Nacional del Perú N²016-17896.

MINSA. (2013). Mortalidad Neonatal en el Perú y sus departamentos 2011 - 2012. Lima.

MINSA. (2016). Situacion epidemiologica de la mortalidad fetal y neonatal, Perú 2016. Lima. 